\title{
Lipidomic analysis of fats and oils - a lot more than just omega-3
}

\author{
Terry K. Smith* \\ Biomedical Sciences Research Centre, The North Haugh, The University, \\ St. Andrews, Fife Scotland, U.K., KY16 9ST
}

*To whom correspondence should be addressed:

Terry K. Smith

Tel: (0)1334-463412

Fax: (0)1334-462593

Email: tks1@st-andrews.ac.uk

\section{Summary}

Edible oils and fats are among the most abundant cooking ingredients in the world, and are an important part of a healthy balanced diet, especially if they are high in omega- 6 and omega- 3 polyunsaturated fatty acids. Rather than just the total fatty acid compositions, the analysis of individual lipid species within these oils and fats have become increasingly important. Within the past decade several mass spectrometric lipidomics methods have been adapted and applied to the analysis of edible oils and fats. These methodologies are vital for the analysis of a plethora of lipid species that will be important for numerous health and sustainable issues in the future.

\section{Introduction}

Edible oils are among the most abundant cooking ingredients through-out the world, varying between culture and country. Some commonly eaten oils include: canola, corn, cottonseed, olive, peanut, safflower, soybean, sunflower oil, while others such as such as walnut oil and sesame oil are used mainly as flavorings. Most oils from plant sources are high in monounsaturated and/or polyunsaturated fats, while low in saturated fats. Solid fats like butter and shortening come from many animal foods or are made by hydrogenation of vegetable oils and these along with coconut and palm, which are naturally high in saturated fats are often used in baking and frying food and for non-cooking products such as salad dressing, margarine spreads, and dips. Numerous oils are used as food supplements for their nutrient content or reported medicinal effect, such as borage, blackcurrant seed, linseed and evening primrose oil. Numerous oils and fats are also utilised in cosmetic products and animal feedstocks. Within the last decade there has been a significant increase in the use of plant crops to produce oils for making biodiesel fuel and fine chemicals as a sustainable alternative to fossil fuels [1 and refs therein].

Depending upon the biological source of the oil or fat, there is an enormous amount of variation in the fatty acid content. In terms of health benefits and the subsequent use of an oil or fat, it is not only in the ratio of saturated to mono- and poly-unsaturated, but also the size (chain-length), and the number, and position(s) of the double bond(s) that is important. Therefore, historically, total fatty analysis by gaschromatography-mass spectrometry (GC-MS) has adequately provided this information and has been the mainstay of the oil and fat community. This has normally been done by base / acid hydrolysis to liberate the fatty acids from whatever lipid, sterol or alcohol they are covalently attached to. Derivatisation into fatty acid methyl esters (possibly further derivatisation/modification of hydroxyl 
groups or double bonds if required) is followed by GC-MS analysis, allowing separation and quantification of each of the individual fatty acid species and isomers.

It is well documented that omega-3/omega-6 polyunsaturated fatty acids (PUFA) are essential for normal growth and development, especially for visual and neurological development in infants. These fatty acids have also been shown to have numerous beneficial effects on various aspects of human health [1]. As humans we are unable to de novo synthesise omega-3 PUFA, as we do not have the necessary fatty acid desaturase enzyme(s) and so rely solely upon dietary intake of these PUFA, with the recommended minimal daily intake being $250 \mathrm{mg}$. Two crucial PUFAs, eicosapentaenoic acid (EPA) and docosahexaenoic acid (DHA), are primarily accessed via marine sources, i.e. fish or krill oil, while $\alpha$-linolenic acid (ALA) is found in numerous plant sources such as nuts and seeds.

However, it is now becoming clear that it is increasingly important to know which fatty acids especially EPA and DHA are attached to which lipid species and how. This has recently become apparent as no significant statistically correlation is observed between omega-3 PUFAs and the reduced risk of cardiovascular diseases (CVDs) when 20 studies on 68680 patients were re-evaluated. The latest evidence suggests the true biologically active component, or parent lipid species, has a direct influence on its delivery and subsequent usage / in vivo activity.

\section{Components of oils and fats}

The primary components ( $90 \%$ ) of natural fats and oils are triacylglycerols (TAGs) and the chemical and physical properties of fats and oils are mainly determined by the fatty acid composition and their position within these TAG molecules [1] (see figure 1). Clinical studies have also shown that the type and position of the fatty acyl substituents of TAGs play an essential role in lipid digestion, absorption and metabolism. Therefore, the development of methods for the positional analysis of individual TAG species can provide valuable information for the planning of dietary, nutritional, and metabolic studies. Other non-polar lipid components of oils are diacylglycerol ( $5 \%)$, monoacylglycerol $(0-5 \%)$, phyosterols and their acyl-esters (0-2\%), and lastly, waxes (0-2\%), which are acyl esters of long chain alcohols. Phospholipids (0-2\%) and free fatty acids (0-1\%) are also present, although their presence is normally ignored. Other minor components include sterol-glucosides, long-chain alcohols, numerous carotenoids, natural phenolic antioxidants / vitamins such as tocopherol and beta-carotene and trace metals, mainly copper, zinc and iron.

During processing/refining dehydration of sterols and monoacylglycerols affords steradienes and glycidyl fatty acid esters, these along with various cxidised lipids. polymerised TAGs, dialkylketones are clear indicators of processing and possible contamination.

With the increasing demand for oils and fats, edible and otherwise, determining their lipid/chemical composition provides a range of valuable information, including its nutritional value and authenticating its origin and quality. The most comprehensive way of achieving this goal is by lipidomic analysis.

\section{Mass spectrometric methods used for lipidomic analysis}

Lipidomic is a fast expanding field of biomedical research and is defined as a systems-level identification and quantitation of pathways and networks of cellular lipids molecular species and their interactions with other lipids, proteins and other moieties in vivo. As such a comprehensive and 
quantitative analysis of the lipid species present in an organism, i.e. its lipidome, is an essential component and requires analytical approaches, in particular mass spectrometry.

Mass spectrometric (MS) analysis of complex lipid mixtures, via soft ionization techniques (matrixassisted laser desorption ionization (MALDI), and electrospray ionization (ESI), were first done in the mid-1990s [2, 3]. The development of lipidomics was based upon ESI-MS and has become the most frequently used MS method in lipid profiling for a wide variety of complex biological samples. Direct-Infusion MALDI or ESI MS-based lipidomics methods are conducted without any pre-separation of lipids. These methods are accurate, highly reproducible, highly sensitive, and less time-consuming than many other methods. Direct infusion into normally nano-source-electrospray ionization-tandem MS-MS (nano-ESI-MS-MS) are often linked to a "shotgun" strategy, which is now routinely used in lipidomics. Here, the power of the MS quadraupoles and fragmentation via various MS/MS modes, product-ion scan (see figure 2), precursor-ion scan, and neutral-loss scan, are utilized to identify individual lipid molecular species [2, 3].

The discrimination of lipid stereoisomers, by direct infusion ESI-MS, is achieved by observing the phenomenon that lyso-form fragment ions corresponding to the neutral loss of fatty acyl moieties attached to the $s n-2$ position, as free fatty acids and as ketenes, exhibited consistently higher intensity than their counterpart ions due to the neutral loss of fatty acyl moieties attached to the $s n-1$ position (see figure 2). However, in comparison with the stereoisomers, the regio-isomers, with differences in the double bonds' positions, are even more difficult to distinguished.

Recent progress in high-resolution $(\geq 100000)$ MS instrumentation, such as LTQ Orbitrap MS47-50 and Fourier transfer ion cyclotron resonance mass spectrometry (FTICRMS), provides the ability to separate lipid ions with the same nominal $\mathrm{m} / \mathrm{z}$ values. When utilised with direct infusion, this allows simultaneous analysis of multiple lipid classes without the need for prior separation and even for extensive MS/MS analysis [2].

Other alternative "soft" ionization techniques used in lipidomics analysis include atmospheric pressure chemical ionization (APCI), atmospheric pressure photoionization (APPI), and desorption electrospray ionization (DESI). While not as versatile as, nor as sensitive as ESI for the analysis of polar lipids, APCI and APPI are good for the nonpolar lipids, with APPI offering higher sensitivity for some lowpolar and nonpolar lipids.

Direct infusion has a couple of disadvantages, firstly, suppression of ionization, in particular in the case of crude extracts and when low-abundance species are to analysed, and secondly, the absolute quantification of lipid species requires considerable efforts (for example, class and mass dependent internal standards). A way around this is by separation of polar/non-polar or lipid classes by liquid chromatography (LC) prior to analysis within the MS. Various automation and integrated methodologies of coupled LC-MS / LC-MS-MS has made direct profiling of complex lipid mixtures easier.

Various LC/MS approaches have been used in lipidomic analysis [2, 3], some of which are listed below. HILIC-LC/ESI-MS - ideal for polar lipid classes, requires optimisation with various column types ( $\mathrm{Si}$, amino or diol) and mobile phases, water content, $\mathrm{pH}$, and salt concentration.

NP-LC/APCI-MS - non-polar lipid classes, Si or amino-columns, mobile phases with hexane, 2propanol or chloroform, allows separation of 6 non-polar lipid classes and 2 regio-isomers of DAGs. $R P-L C / E S I-M S$ - all lipid species, $\mathrm{C} 18$ and $\mathrm{C} 8$ columns, mobile phases typically aqueous/acetonitrile 
mixtures

NARP-LC/APCI-MS - non-polar lipid species, C18 columns, mobile phases typically propan-2-ol, acetonitrile, acetone or dichlormethane

Ag-LC/APCI-MS - non-polar lipids species, ion-exchange column with $\mathrm{Ag}^{+}$, mobile phases typically hexane-acetonitrile/2-propanol or dichlormethane-acetonitrile mixtures.

Chiral-LC/APCI-MS - non-polar lipid species, chiral columns, normal phase mobile system with hexane and propan-2-ol

NARP-LC/APCI-MS of Nonpolar Lipids - non-polar lipids, e.g. TAGs, DAGs, wax esters, cholesterol and its esters, the separation of TGs is according to their Equivalent carbon number which is equal to the carbon number in all acyl chains- $2 *$ double bond number.

\section{TAG analysis}

RP-LC/MS and RP-LC/MS/MS are commonly employed for comprehensive TAG analysis and have been employed successfully with various vegetable oils and animal fats. However, RP-HPLC typically cannot separate positional isomers of TAGs (i.e. AAB and ABA). Therefore, the MS is utilized to exploit the differential fragmentation of the fatty acid at the $s n-2$ position relative to the loss of the fatty acids at the $s n-1$ and $s n-3$ positions.

APCI has been utilised successfully to allow in-source fragmentation, generating the DAG fragments, for the identification of TAG positional isomers. However, for complex mixtures with potential coeluting TAGs this has proven problematic. Various alternative ionisation techniques and methodologies have been tried for the determination of the position of fatty acids in TAGs, including ESI. Despite ESI not being considered the ideal ionization mode for non-polar lipids, it has been successfully employed for the determination of TAG positional isomers.

Herrera et al [4] reported using RP-ESI-MS/MS for the quantification of TAG regioisomers in sunflower and olive oils. Under low-energy collision-induced dissociation conditions, fragmentation was obtained, that was dependent on the nature and position of the acyl substituents on TAG. They were observed for four pairs of 'AAB/ABA'-type TAGs, namely PPO/POP, OOP/OPO, LLO/LOL and OOL/OLO (where $\mathrm{P}$ is 16:0, palmitic acid, $\mathrm{O}$ is 18:1, oleic acid, and $\mathrm{L}$ is $18: 2$, linoleic acid). For the $[\mathrm{M}+\mathrm{Na}]^{+}$adducts, the loss of the fatty acid in the outer positions ( $s n-1$ or $\left.s n-3\right)$ was favored over the loss in the central position (sn-2), which enabled the determination of the fractional abundance of the isomers. Ratios of the intensity of fragment ions at various AAB/ABA compositions produced linear calibration curves with the $[\mathrm{M}+\mathrm{Na}]^{+}$adducts, providing a consistent level of isomeric discrimination for the TAGs studied. It also offered the most convenience, in that they required no additive to the mobile phase.

\section{Wax esters}

Wax esters are a class of highly hydrophobic neutral lipids occurring in a variety of oils, including oilseed rape, where it is seen as a sustainable source for this high value chemical. Previous analyses have involved prior separation and APCI-MS or GC-MS analysis. Recently, a direct-infusion nano-ESIMS/MS method has been developed for fast and semi-quantitative determination of wax ester compositions from complex lipid mixtures, relying upon targeted multiple reactant monitoring detection of intact wax ester species [5].

Lipidomic analyses of sterols, sterol-esters, and glycidyl fatty acid esters has also been successfully 
done using direct-infusion "shotgun" methodologies using nano-ESI-MS-MS $[2,3,6]$. This can be done with the same sample and scan modes as described earlier, thus saving time and sample as separate GCMS analyses are not required.

\section{Data processing}

Very often as with all "omics" platforms, it is the interpretation and processing of the enormous amounts of data that is the rate limiting-step. This is particulary so when you consider the numerous possibe individual lipid species, including isomers there could arise from a single mass peak. I believe those of us who do lipidomic analysis and interpretation should be given far more credit from other "omic" communities, especially as the current estimates is for 3000-7500 individual lipid species in a typical eukaryotic cell. Thankfully there are some software packages available to help, including some dedicated to lipidomics, LipidView, Lipid Search and TransOmics. There are also many web-based databases and open source tools available, such as http://www.lipidmaps.org/, http://lipidlibrary.aocs.org/, http://www.cyberlipid.org/, http://www.lipidbank.jp/, https://wiki.mpicbg.de/lipidx/, http://www.mmass.org/ and http://mzmine.sourceforge.net/.

\section{Conclusions}

The advancement in mass spectrometry and its application to lipidomics in the past twenty years has enlightened us with the full appreciation of lipid heterogeneity in biological systems has spurred a new era in lipid biology. With the increasing demand for high-value lipid entities such as omega-3 and -6 PUFA and waxes, from the ever-decreasing marine and fossil oil resources respectively, there will be numerous opportunities for lipid based research and development to generate these species via food crops or other biological systems a sustainable alternatives.

\section{Acknowledgements}

TKS research is supported in part by the Wellcome Trust, SUSLA, BBSRC and the European Community's Seventh Framework Programme under grant agreement No.602773 (Project KINDRED). TKS is also an academic consultant for Mylnefield Lipid analysis and Lamellar Biomedical Ltd.

\section{References}

1. M E. Carrin and A A. Carelli (2010) Peanut oil: Compositional data Eur. J. Lipid Sci. Technol. 112, 697-707

2. Min Li, Li Yang, Yu Bai, and Huwei Liu (2014) Analytical Methods in Lipidomics and Their Applications Anal. Chem. 86, 161-175

3. Markus R Wenk (2010) Lipidomics: New Tools and Applications Cell 143, 888-895

4. Lisandra Cubero Herrera, Michael A. Potvin and Jeremy E. Melanson (2010) Quantitative analysis of positional isomers of triacylglycerols via electrospray ionization tandem mass spectrometry of sodiated adducts Rapid Commun. Mass Spectrom.; 24: 2745-2752

5. Tim Iven1, Cornelia Herrfurth, Ellen Hornung, Mareike Heilmann, Per Hofvander, Sten Stymne, Li-Hua Zhu and Ivo Feussner (2013) Wax ester profiling of seed oil by nano-electrospray ionization tandem mass spectrometry Plant Methods, 9:24

6. Becalski A1, Feng SY, Lau BP, Zhao T (2012) Glycidyl fatty acid esters in food by LC-MS/MS: method development. Anal Bioanal Chem. 403(10): 2933-42. 


\section{Figure legends:}

Figure 1: Lipidomic analysis peanut oil. Positive ion ES-MS survey scans $(600-1000 \mathrm{~m} / \mathrm{z})$, shows a heterogeneous mixture of TAGs and DAGs and minor phosphatidylcholine components in the lipid extract. Annotation of the major lipid species, based upon daughter ion fragmentation analysis by ESMS-MS. Sample analysed on a triple quadrupole mass spectrometer (Absceix 4000 QTrap) equipped with a nanoelectrospray source. Samples were delivered into the spectrometer using a Nanomate interface in direct infusion mode $(\sim 125 \mathrm{~nL} / \mathrm{min})$.

Figure 2: Fragmentation of ESI-MS-MS (A) Schematic of triple quadrapole and the fragmentation. (B) Negative daughter ion fragmentation analysis by ES-MS-MS of $835.5 \mathrm{~m} / \mathrm{z}(200-900 \mathrm{~m} / \mathrm{z})$. Structural representation of the fragments ion obtained is given below lipid species.

\section{FIG 1}

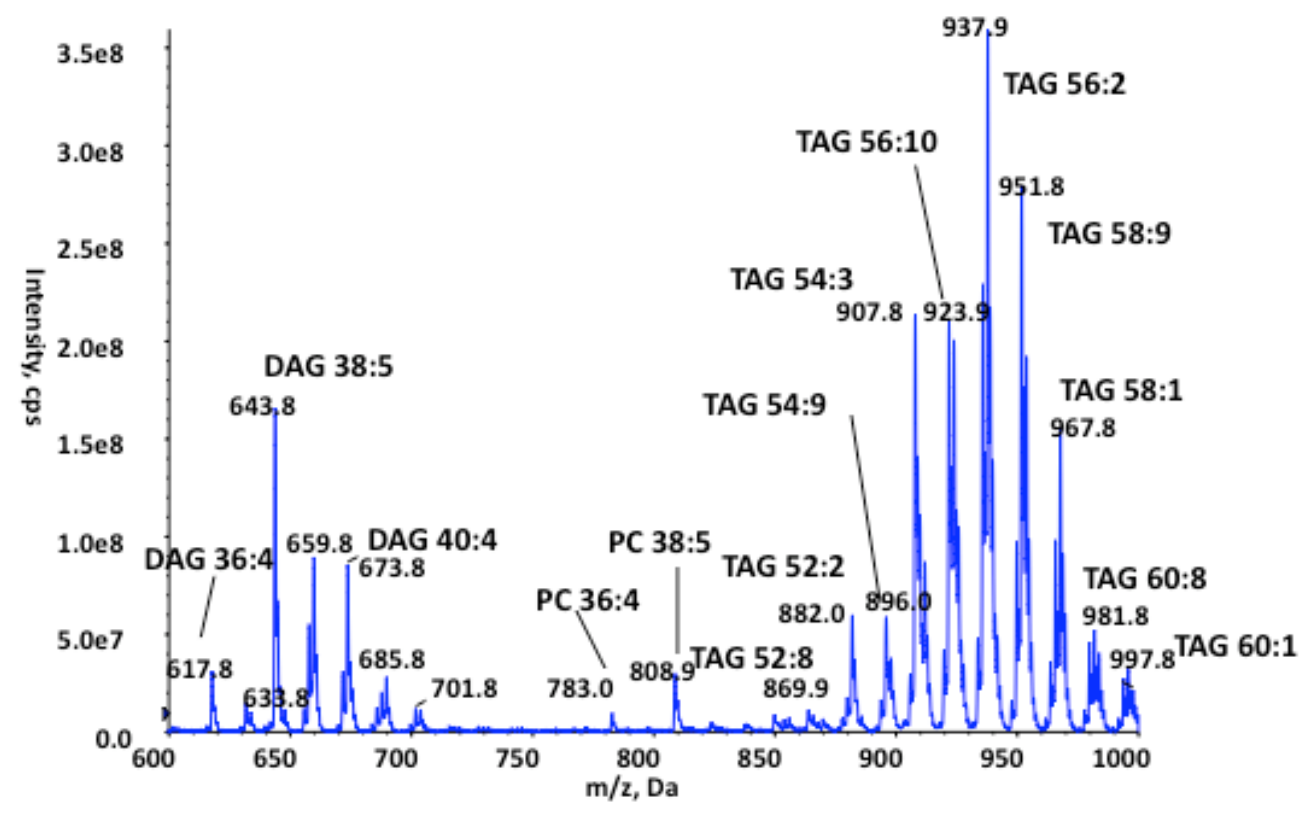




\section{FIG 2}

A

- Specific $[\mathrm{M}-\mathrm{H}]^{-}$ion

- Non-specific ions

- Fragment ions

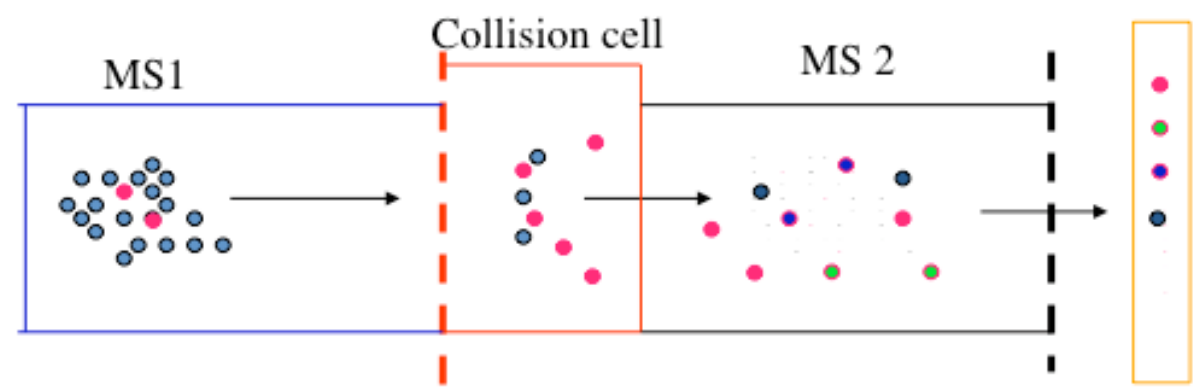

Only ions of one mass $(835.5 \mathrm{~m} / \mathrm{z}$ ) are collided All fragment ions reach detector

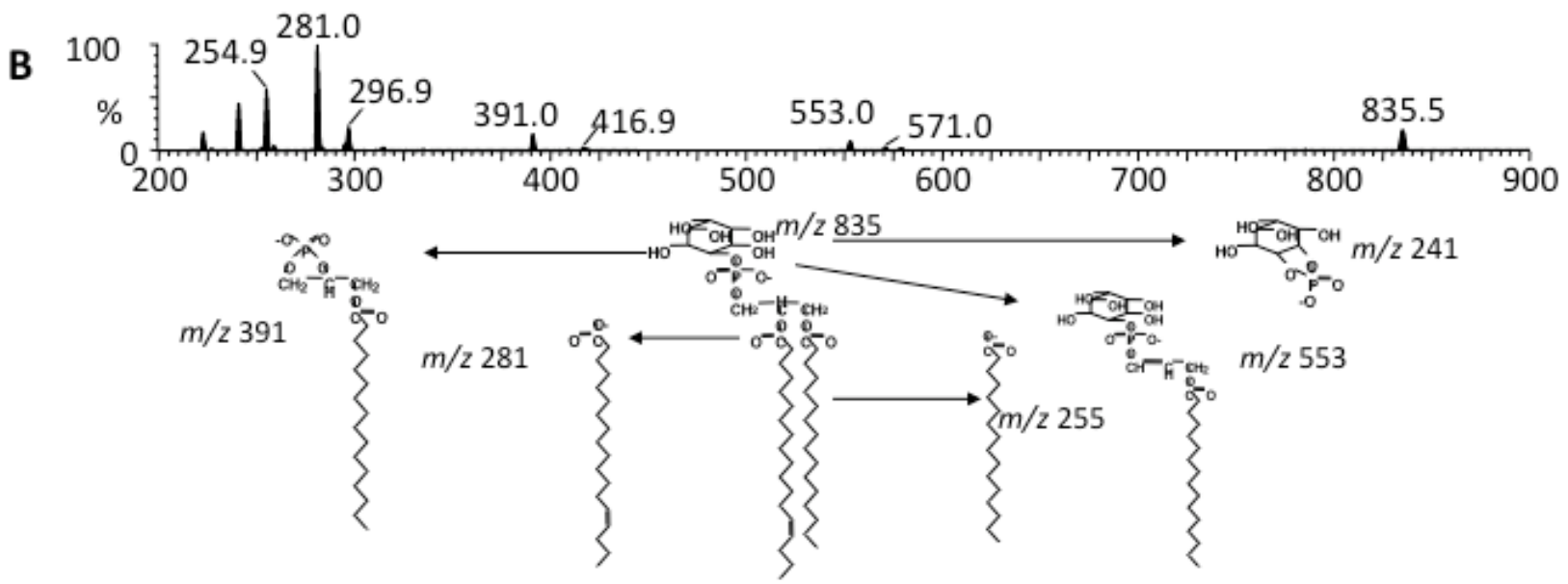

$835.5 \mathrm{~m} / \mathrm{z}$ PI sn-1 C16:0, sn-2 C18:1 